Infusionstherapie 1990;17:359-362

\title{
Jahres-Inhalt 1990
}

\section{Übersichtsarbeiten}

Adolph, M.,' Eckart, J. (Augsburg)

Der Energiebedarf operierter, verletzter und septischer

Patienten 5

Bäßler, K.H. (Mainz)

Die Bedeutung der Vitamine in der parenteralen

Ernährung

19

Björntorp, P. (Göteborg)

Adipositas und Verteilung des Tettgewebes als Risikofaktoren

für die Entwicklung von Krankheiten 24

Garrow, J. S. (London)

Wie beugt man Übergewicht vor? 28

Originalarbeiten

Behrendt, W,; Bogatz, V.; Giani, G. (Aachen)

Der Einfluß der posttraumatischen Kalorien- und Stickstoffzufuhr

auf die kumulative Stickstoffbilanz 32

Pohlandt, F. (Ulm), Wagner, M. (Stuttgart), Rhein, R. (München),

Obladen, M. (Berlin)

Eine neue Aminosäurenlösung für die parenterale Ernährung

von Frühgeborenen, Neugeborenen und Säuglingen 40

Albert, S.; Seidl, S.; Doerr, H.W. (Frankfurt)

Kühnl, P.; Sibrowski, W. (Hamburg)

Die Bedeutung des Zytomegalievirus im Blutspendewesen 48

Kongreßberichte 58

Mitteilungen der Deutschen Gesellschaft für

Transfusionsmedizin und Immunhämatologie

DAKE-Mitteilungen 60

Übersichtsarbeiten

Hayde, M.; Widhalm, K. (Wien)

Kohlenhydrate in der Therapie von Glykogenosen 69

Originalarbeiten

Glück, D.; Kubanek, B.; Elbert, G.; Gathof, B.; Gräßmann, W.;

Hesse, R.; Holzberger, G.; Koerner, K.; Vornwald, A. (Ulm)

Risiko einer HГV-Infektion durch frühere Blutspenden von

Spendern, die bei Routine-Tests in Blutbanken als HIV-

Antikörper-positiv erkannt wurden. Retrospektive Studie in

Blutbanken des Deutschen Roten Kreuzes 73

Kortsík, C. St. F.; Staedt, U.; Stein, T,; Geiger, G.;

Buss, J. (Mannheim) 
Konjunktivaler Sauerstoffpartialdruck, Hämorheologie und

Kreislaufparameter bei akutem zerebralem Insult vor und nach

Infusion von $\beta$ prozentiger niedermolekularer

Hydroxyäthylstärke 79

Prinzler, H.-J. (Braunfels); Weidler, B.;

Lohmann, B. (Gießen); Bormann, B. von (Duisburg)

Routinemäßige postoperative parenterale Ernährung mit einer

Komplettiösung $\quad 84$

Butters, M.; Bittner, R.; Engst, U.; Roscher, R.;

Beger, H. G. (Ulm)

Der Einfluß der Leberpassage auf die peripheren Glukose- und

Hormonkonzentrationen bei friihpostoperativer enteraler

Ernährung $\quad 89$

Haupt, W.; Husemann, B.; Sailer, D. (Erlangen)

Postoperative parenterale Ernährung nach

Lebersegmentresektion - sind Fettemulsionen ein Risiko? .... 94

Figueras, J.; Ramos, E.; L·lop, J. M.; San-Juan, N.;

Marti, J. (Barcelona)

Effekt einer ausgewogenen Aminosäuren-Lösung auf den

Proteinstoffwechsel nach operativer Behandlung. Eine klinische

Studie 100

Meraihi, Z.; Lutz, O.; Scheftel, M.; Frey, A.;

Bach, A. C. (Strasbourg)

Gramnegative Sepsis bei der Ratte und Aktivität der

Lipoproteinlipase bei LCT- und MCT/LCT-haltigen,

kommerziellen parenteralen Emulsionen 104

Hypothese

Ravussin, E.; Bogardus, C. (Phoenix, AZ)

Energieaufwand bei Adipositas: Gibt es einen «sparsamen

Erbfaktor»? 108

DAKE-Mitteilungen 113

Übersichtsarbeiten

Ollenschläger, G.; Schrappe-Bächer, M.; Fätkenheuer, G.;

Salzberger, B.; Burger, B.; Kaufmann, W. (Köln)

Mangelernährung - ein klinisch relevantes Problem der HIV-1-

Infektion?

119

Bray, G.A. (Los Angeles)

Endokrine Störungen und verminderte Aktivität des

sympathischen Nervensystems bei der Entwicklung der Fettsucht 124

Sachs, V.; Washington, G. (Kiel)

Die präoperative Eigenblutspende

131

Originalarbeiten

Hankeln, K.; Senker, R.; Beez, M. (Bremen)

Vergleichende Untersuchung zur intraoperativen Wirksamkeit

von $5 \%$ Humanalbumin oder $10 \%$ Hydroxyäthylstärke

(HAES-steril@) auf Hämodynamik und Sauerstofftransport 
bei 40 Patienten

135

von Bormann, B.; Idelberger, R. (Duisburg),

Sticker, J.; Ratthey, K.; Hempelmann, G. (Gießen)

Yolumensubstitution während akuter normovolämischer

Hämodilution (ANH) - Humanalbumin $5 \%$ vs.

Hydroxyäthylstärke 6\% 142

Schroder, F. (Bremen)

Partikelbelastung in der Intensivtherapie. Lösungsmöglichkeiten durch Multilumen-Katheter und Intrapur-1' -Filter 149

Schütt, K.-H.; Pietsch, M.; Schicketanz, K.-H. (Mainz)

Screeningverfahren zur Bestimmung von Tetanus-

Antikörperkonzentrationen in Blutprodukten 156

Glück, D.; Kubanek, B.; Gaus, W. (Ulm)

Aktuelle Daten zur Prävalenz und Epidemiologie des HIV aus

der HIV-Studie der DRK·Blutspendedienste der BRD

Hönlinger, M.; Klima, G.; Schönitzer, D. (Innsbruck)

Partikelbildung und Analyse während einer simulierten

Thrombozytapherese 164

360

Jahres-Inhalt 1990

Laufende klinische Studien

Albert, A.; Wendt, M.; Reiffer, B. (Münster)

Klinische Erfahrungen mit zentral-venösen Multilumen-

Kathetern 168

Mitteilungen der Deutschen Gesellschaft für

Transfusionsmedizin und Immunhämatologie

Sonderbände

154

Veranstaltungskalender

Informationen für die Klinik

227

Erratum

230

Mitteilungen der Deutschen Arbeitsgemeinschaft für künstliche

Ernährung (DAKE) 231

MEDICA-Programm 210

Buchbesprechungen 229

Originalarbeiten

Fauth, U.; Heinrichs, W.; Puente-Gonzalez, L; Halmágyi, M.

(Mainz)

Maximale Umsatzraten an Enzymen der Glykolyse und des

Zitratzyklus von separierten Granulozyten in der postoperativen

Phase 178

Herrmann, A.; Jauch, K.W.; Günther, B.; Schildberg, F.W.

(München)

Elimination und Stoffwechsel MCT-haltiger Fettemulsionen am operierten Patienten im Rahmen einer vollständigen 
parenteralen Ernährung

185

Hampl, J. (Erlangen), Matzkies, F. (Bad Neustadt/Saale)

Maltose-Dextran - Eine neue Kombinationslösung zur

Induktion einer Diurese beim Menschen 197

Fuchs, M.; Henkel, P.; Becker-Scheid, D.; Schmitt, J. (Losheim)

Kontinuierliche Hämodilution mit einer niedrigmolekularen

Hydroxyaethylstärkelösung. Pilotuntersuchungen bei Patienten

mit akuter zerebraler Ischämie 205

Dietrich, G.; Kretschmer, V.; Orth, D.; Haupt, W. (Marburg)

Primäre Hämostase bei Hämodilution-1) Hämatokrit 212

Dietrich, G.; Orth, D.; Haupt, W.; Kretschmer, V. (Marburg) Primäre Hämostase bei

Hämodilution-2) Infusionslösungen. . . 214

Giannitsis, D.J.; Flierl, D.; Schuler, G.; Häcker-Shahin, B.

(Homburg)

Nachweis von Blutgruppenantikörpern mittels ELISA unter

Einsatz von Erythrozytenstroma 217

Glück, D.; Wiedeck, H.; van Wickern, M.; Wölpl, A.;

Northoff, H.;Ahnefeld, F.W.; Grünert, A.; Kubanek, B. (Ulm)

Anti-Lipopolysaccharid-Immunglobulin-Therapie

(IgG-Anti-LPS) bei Intensivpatienten nach Operation

bei Infektion 220

Prävention

Jäger, H.; Rauhaus, G.; Peyerl, G.; Korte, R. (Eschborn),

Nseka, K.; Goussard, B.; Kabeya, C.-M.; Salaun, J.-J.

(Kinshasa, Zaire)

Blutspenderwerbung als Mittel zur Verminderung der

Übertragung von HIV-1, Hepatitis B und Syphilis in Kinshasa,

Zaire 224

Editorial

Fürst, P.; Stehle, P. (Stuttgart)

Künstliche Ernährung -gestern, heute, morgen 237

Originalarbeiten

Calon, B.; Pottecher, 71; Frey, A.; Ravanello, J.; Otteni, J. C;

Bach, A. C. (Strasbourg/France)

Langkettige versus mittel- und langkettige Triglyzerid-

Fettemulsionen in der parenteralen Ernährung von Patienten

mit Schädel-Hirn-Trauma 246

Fässle, V.; Ahnefeld, F. W.; Grünert, A. (Ulm)

Vergleichende Untersuchungen zur Applikation von Xylit oder

Glukose bei der parenteralen Zufuhr von Aminosäuren bei

¡ntensivmedizinischen Patienten 251

Butters, M.; Miller, W.; Bittner, R. (Stuttgart)

Der Einfluß einer enteralen und parenteralen Ernährung auf die

Glukosetoleranz in der friihpostoperativen Phase 257

Kreinhoff, U. (Gießen), Elmadfa, I. (Wien),

Salomon, F. (Lemgo), Weidler, B. (Gießen) 
Untersuchungen zum Antioxidantienstatus nach operativem Streß 261

Jung. F.; Koscielny, J.; Mrowietz, C. (Homburg/Saar),

Wolf, S. (Aachen), Kiesewetter, H.; Wenzel, E. (Homburg/Saar)

Einfluß der Hämodilution auf den systemischen und den

Kapillarhämatokrit 268

Lackner, F. X.; Graninger, W.; Bias, W.; Panzer, S.;

Schulz, E. (Wien)

Präoperative Eigenblutspende, der Einfluß von

Hydroxyäthylstärke auf Retikuloendothelialsystem und

Opsonine 276

Wittkopf, D.; Grundmann, A.; Sibrowski, W.;

v. Eisenhart-Rothe, B.; Kühnl, P. (Hamburg)

Analyse irregulärer Antikörper in der Abteilung

für Transfusionsmedizin am Universitäts-Krankenhaus

Hamburg-Eppendorf von 1984-1988 280

Mitteilungen der Deutschen Arbeitsgemeinschaft für künstliche

Ernährung (DAKE) 283

Mitteilungen der Deutschen Gesellschaft für

Transfusionsmedizin und Immunhämatologie (DGTI)

Erratum 285

Inhaltsverzeichnis 234

Sonderbände 275

Jahres-Inhalt 1990

361

Übersichtsarbeiten

Richter, G.; Dehnert, J.

Sondenernährung in der Onkologie 291

Originalarbeiten

Günther, H.J.; Saeger, H.D.; Hohner, E.; Striebel, J.P.;

Scigalla, P.; Bartels, F.; Reichardt, I.

Peripher-venöse bedarfsadaptierte parenterale Ernährung:

Renaissance der Maltose? 300

Druml, W.; Zadravec, S.; Kerbl, H.; Grimm, G.; Schneeweiß, B. Intensivmedizinischer Einsatz einer neuen Fettemulsion 306 Penner, M; Fíngerhut, D.; Tacke, A.

Einfluß einer neuen Hydroxyäthylstärkelösung 10\% HES/270/0,5 auf Blutgerinnung, Blutverlust und Hämodynamik im Vergleich zu PPL 3,5\% 314

Baak, L.C.; Jansen, J.B.M.J.; Lamers, C.B.H.W.

Effekt von intravenösen Aminosäuren auf intragastrale

pH-Werte während ambulanter pH-Metrie 321

ABSTRACTS der 22. Jahrestagung der

Deutschen Arbeitsgemeinschaft für Histokompatibilitätstestung,

Hamburg, 4.-6. Oktoberl990 326 
Informationen für die Klinik

Ausschuß für Nährstoffbedarf der DAKE:

L-Tryptophan in Infusionslösungen 352

Mitteilungen der Deutschen Gesellschaft für Transfusionsmedizin

und Immunhämatologie (DGTI) 353

Editorial 289

Buchbesprechungen 352

Autorenverzeichnis $1990 \quad 358$

Jahresinhalt $1990 \quad 359$

Sachwortverzeichnis 363

362

Jahres-Inhalt 1990

Supplement 1

Abstracts

zur 9. geme;nsamen Jahrestagung der

Österreichischen Arbeitsgemeinschaft für

klinische Ernährung (AKE)

und der

Deutschen Arbeitsgemeinschaft für

künstliche Ernährung (DAKE)

Wien, 28.-31. März 1990

Supplement 2

Editorial 3

Groh, /.; Dieterich, H.-J.; Peter K. (München)

Hypovolämisch-hämorrhagischer Schock,

Pathophysiologie und Therapie 4

Kretschmer, V. (Marburg)

Perioperative Gerinnungstherapie und -diagnostik 9

Zander, R. (Mainz)

Der arterielle Sauerstoff-Status als limitierender Faktor einer

Hämodilution 20

Klövekorn, W. P. (München)

Die myokardiale Sauerstoffversorgung unter Hämodilution bei

herzgesunden und -chirurgischen Patienten 24

Mehrkens, H,-H.; Geiger, P.; Weindler, M.; Wollinsky, K.H.;

Pohland, H. (Ulm), Schleinzer, W. (Hamburg)

Vier Jahre Erfahrung mit dem autologen Transfusionskonzept

Ulm (ATU) 28

Heim, M.U.; Mempel, W. (München)

Einsatz von FFP im operativen Bereich unter besonderer

Beriicksichtigung des Eigen-FFP 34

Partecke, G. (Memmingen)

Organisation und Praktikabilität des autologen Blutersatzes

im chirurgisschen Bereich eines Krankenhauses der

Versorgungsstufe III 37 
Faul, P.; Partecke, G. (Memmingen)

Der autologe Blutersatz in der Urologie $\quad 41$

Decker, K.; Heeg, P. (Tubingen)

Mikrobiologische Untersuchungen bei intra- und postoperativer

Autotransfusion in der Orthopädie 43

Höhne, M, (Vogtareuth)

Eigenblutspende mit Tíefkühlkonservierung 46

Supplement 3

Foreword 3

Heymsfield, S.B.; Lichtman, S. (New York, NY) New Approaches to Body Composition

Research: A Reexamination of Two-Compartment Model Assumptions .... 4

Shizgal, H.M. (Montreal)

Nuritional Assessment with Body Composition Measurements

by Mulitple Isotope Dilution 9

Hill, G.L. (Auckland)

Clinical Body Composition Using in vivo Neutron Activation

Analysis 18

Burkinshaw, L.; Hedge, A.P.; King, P.F.J.G. (Leeds);

Cohn, S.H. (Upton, NY)

Models of the Distribution of Protein, Water and Electrolytes

in the Human Body 21

Fürst, P. (Stuttgart)

Direct Biochemical Analyses of Human Muscle Tissue 26

Viell, B.; Kill, H.; Vestweber, K.-H.; Rosier, H. (Köln)

Human Muscle Tissue Obtained Intraoperatively from Surgical

Patients - a Tool for the Assessment of Biochemical Body

Composition 35

Bachert-Baumann, P.; Gückel, F.; Semmler, W.; Lorenz, W.J.

(Heidelberg)

Principles of in vivo Magnetic Resonance Spectroscopy in

Whole Body Magnetic Resonance Systems 39

Gückel, F.;Bachert-Baumann, P.;Semmler, W.;Lorenz, W.J.;

Kaick, G. von (Heidelberg)

MR Tomography and Multinuclear MR Spectroscopy in a

Whole Body MR System - Applications in Cancer Research

Heymsfield, S.B. (New York, NY)

Anthropometric Measurements: Application in Hospitalized

Patients 48

Schmitt, R.M. (St. Ingbert)

Ultrasonic Tissue Characterization: Its Potential for the

Estimation of Body Masses 52

Böhm, D.; Wünsch, K.jBaum, M.; Odaischi, M. (Kaiserslautern)

The Ultrasonic Measurement of Upper Arm Muscle and Fat Area 54

Kuhn, C; Holm, E.; Leweling, H.; Barth, H.-O.; Hagmüller, E.

(Mannheim)

How are the Main Indicators of the Nutritional State Interrelated? 56 
Burkinshaw, L. (Leeds)

Some Aspects of Body Composition in Cancer

Nürnberger, H.R.; Löhlein, D. (Dortmund)

Malnutrition in Cancer Patients

59

Fear on, K.C.H. (Edinburgh); Preston, T. (Glasgow)

Body Composition in Cancer Cachexia 63

Shizgal, H.M. (Montreal)

Validation of the Measurement of Body Composition from

Whole Body Bioelectric Impedance 67

Böhm, D.; Odaischi, M.; Beyerlein, Ch.; Overbeck, W.

(Kaiserslautern)

Total Body Water: Changes during Dialysis Estimated by

Bioimpedance Analysis

75

Hill, G.L. (Auckland)

Some Implications of Body Composition Research for Modern

Clinical Management 79 